\title{
Characterization of physical, mechanical, thermal and morphological properties of agro-waste betel nut (Areca catechu) husk fibre
}

\begin{abstract}
This paper investigates the physical, thermal, mechanical and morphological properties of betel nut husk fibre, in order to assess their suitability as lignocellulosic reinforcement for polymer composites. Betel nut husk (BNH) fibres of three different stages of maturity were evaluated to study the effect of fibre maturity on the thermal, physical and mechanical properties of $\mathrm{BNH}$ fibre. The thermal stability of $\mathrm{BNH}$ fibre was studied using the thermogravimetric analysis (TGA) technique. It was found that the thermal stability of the $\mathrm{BNH}$ fibre is influenced by the maturity of the BNH fibre due to the different amounts of cellulose, hemicellulose, lignin, and moisture in the BNH fibre at each stage of maturity (raw, ripe, matured). The BNH fibres showed decrease in fibre length and fibre diameter, and increase in density with the increase in fibre maturity. SEM micrographs of BNH fibre surface revealed the existence of rough and perforated surface of BNH fibre. Whereas, the cross-sectional of the BNH fibre showed that the raw BNH fibres were observed with bigger lumen, whilst ripe BNH fibre exhibits a slightly smaller and elongated lumen. In contrast, matured BNH fibre showed more compact structures instead of hollow-like lumen structures. In terms of mechanical properties, the $\mathrm{BNH}$ fibre tensile properties were found to be comparable to coir and kenaf fibre, which have been widely used as reinforcement in polymer composites.
\end{abstract}

Keyword: Betel nut husk fibre; Natural fibre; Agro-waste; Thermal degradation; Mechanical properties; Polymer composites 